\title{
Inspección técnica, sistema educativo e innovación
}

\author{
AlVARO BUJ GIMENO \\ Universidad Complutense
}

\begin{abstract}
SUMMARY.-This article corresponds to the report presented by the author in the III European Symposium of Educational Supervision, held in Benalmádena (Málaga), from March 30 to April 4, 1992.

It deals with the necessity to establish some principles of definition of the Supervision, and a design of the training program for supervisors. This objective is basec upon a systemic understanding of Education, which results in an investigation about the functions, roles and skills required for the supervisors, in order to improve their consideration as professionals.

Later on, the article analyzes the norms of the Educational Supervision in Spain, which permits to know the administrative requirements in this field.

Finally, the union made up by the theoric model of Supervision and the description of the supervisor -required by the current Spanish educational policy-, is connected with the educational change encouraged in Spain. All this leads to the conclusion that the Supervision must have a set of competences in order to intervene efficiently and professionally in the so called educational reform.
\end{abstract}

\section{INSPECCIÓN TÉCNICA}

La consideración previa a un intento de definición de inspección educativa sería qué criterio vamos a adoptar para llegar a ella de un modo metodológicamente correcto. Podemos atenernos a su evolución histórica, considerar qué es lo que hacen los Inspectores, estudiando cuáles son los requisitos de su formación y selección o quizá analizando las funciones que los responsables de la política educativa les adjudican y las competencias que les otorgan para el desarrollo de aquéllas.

Entiendo que dichos métodos de análisis pueden ser calificados de parciales, es decir, faltos de una visión más comprensiva. Desde que los investigadores de la teoría de la ciencia, partiendo de modelos de comportamiento biológico, introdujeron la teoría de sistemas los distintos campos del saber se han beneficiado, tanto en lo teórico cuanto en lo práctico, de dicha concepción científica. También la educación puede ser estudiada como un sistema y abordado tecnológicamente, es decir, como una combinación ordena- 
da de partes que, aunque puedan aparecer de manera independiente, interactúan entre sí y constituyen un todo racional, funcional y organizado, a fin de alcanzar metas previamente definidas. A su vez el sistema educativo forma parte del sistema social, mejor dicho, es un subsistema de éste último. Actualmente en Europa se avanza hacia un sistema social globalizador, pasando de la organización cerrada de los Estados que lo integran hacia una estructuración intereuropea; ésta sería una razón mayor para avanzar en la definición de inspección educativa.

Otro matiz a tener en cuenta es que los sistemas se dan en distintos niveles. De esta forma los niveles superiores del sistema educativo pueden integrar otros niveles del propio sistema, apareciendo, como veremos más adelante, la necesidad de la presencia de la inspección en todos los niveles.

En el aspecto terminológico cabe asimismo alguna otra consideración. Evidentemente la ciencia avanza gracias a la metodología y en la medida en que su terminología alcanza precisión, univocidad y universalidad. Ciertas matizaciones pueden ser hechas; por ejemplo, admitida la educación como sistema es necesario superar la denominación inspección escolar y pasar a la de mayor contenido cual es la de inspección educativa.

Otra consideración cabe hacer sobre las denominaciones, supervisión e inspección; para distinguirlas se alude al énfasis que cada una pone en alguna de las funciones específicas desempeñadas por los profesionales; parece que inspección se liga más a tareas administrativas y la supervisión a las docentes. No obstante también está en uso el apelativo germánico de consejero escolar, por la singularidad dominante de su función asesora. Si de estilos, papeles o actitudes se trata, teniendo en cuenta el modo de ejercer las actividades profesionales se ha aludido a inspección autocrática, liberal y democrática; dichas distinciones no responden a la esencia de la profesión, sino al contexto políticosocial en que se ejerce.

Para superar todo tipo de connotaciones parciales, bien sean de denominación o conceptuales, es necesario distinguir las funciones en sí mismas, de las que, especialmente, en tiempo y lugar realice el inspector. La definición profesional viene caracterizada en la configuración de la educación como sistema, es decir, por los papeles desarrollados y competencias necesarias para intervenir en la situación de entrada, proceso, producto, ambiente y realimentación del sistema. La definición accidental proviene de aquéllo que, en tiempo y lugar realice concretamente el inspector por imperativo de otro «sistema» que es el diseñado por la administración pública concordante con una determinada concepción ideológica de la educación. Una tarea global y singularmente profesional puede ser la que realice la inspección diagnosticando los problemas y asesorando sobre los modos de corregirlos, por ejemplo, en una institución educativa, para hacer que alcance los objetivos propuestos, ésta sería una labor de auditoría. No obstante todos sabemos del marco habitual en que la inspección trabaja dado que, en un Estado de derecho, el sistema educativo funciona para prestar y garantizar el derecho a la educación que asiste a los ciudadanos, razón por la cual se entiende la vinculación predominante de la Inspección a la función pública; ésto explica la habitual dependencia de la Inspección respecto de la política educativa. Sin embargo es necesario insistir en la distinción precedente, ya que si no se hiciera, la formación profesional del inspector puede ser circunstancial, cambiante y dependiente de pautas ideológicas mas que de las propiamente nomotéticas, lo cual conlleva el riesgo de que se le proporcione una formación carente de rigor científico. 
Cuanto antecede justifica la estructura y partes de esta exposición, que, pretende abordar siquiera sea sucintamente, la capacitación profesional del Inspector de educación, los papeles que desempeña en el seno de la educación como sistema, las funciones que le asignan las normas emanadas de la Administración pública y la relevancia de su tarea en la toma de decisiones necesaria para toda innovación educativa.

\section{Concepción sistémica de la educación e inspección técnica}

Desde que Bertalanffy (1950) aportó el concepto de teoría de sistemas no solamente la biología, sino también las ciencias humanas, entre las que contamos a la ciencia de la educación, se han beneficiado de esta revolución científica.

Ciertas premisas de los sistemas analítico-mecanicistas han quedado sustituidas por las de la teoría de sistemas; así, la de separabilidad de los componentes por la de una patente irreductibilidad; la del equilibrio por la de resistencia al desorden; la del todo como suma de partes por la de que aquél puede ser más que la suma de sus partes; finalmente, que las propiedades de las organizaciones no se pueden deducir de las de sus componentes.

En el caso del sistema educativo parecen generalmente admitidos los siguientes componentes: finalidades, educandos, educadores, órganos de dirección, contenidos educativos, material didáctico, tecnología educativa, centros, planes de estudios, horarios, controles cuantitativos y cualitativos, aspecto investigador, aspecto económico y aspecto administrativo. Cuando los elementos mencionados se aplican a organizaciones complejas es preciso reconocer, además, los distintos niveles jerárquicos de funcionamiento del sistema; ésto representa una serie de interconexiones en que el control y la regulación de un sistema interno constituyen el control interno y la regulación de otro; precisamente en educación se pueden observar jerarquías verticales de autoridad o de actividades profesionales. Al analizar la estructura que dichos elementos presentan es importante no sólo su estudio sino la determinación específica de las conexiones, dentro del mismo, que son formadoras del sistema, o sea que garantizan la unidad de éste en el transcurso de todas sus posibles modificaciones y alteraciones. Como relaciones formadoras hay que destacar las más específicamente cualitativas del sistema, como son la autorregulación interior y sobre todo los mecanismos que determinan la dirección del desarrollo del mismo; entre ellos está la meta interior del desarrollo, objetivo que diferencia a un sistema orgánico de la simple suma de elementos.

En esta sucinta justificación del planteamiento educativo tampoco podemos dejar de citar algunos conceptos para aludir al funcionamiento de un sistema. Así, los propósitos que representan el estado que el sistema pretende alcanzar, ajustado a las exigencias normativas y reglamentarias, que son propios del sistema. Las metas, que son externas al sistema educativo, por ejemplo, el estado de la educación de un país en relación con las tendencias de la sociedad. El sistema en funcionamiento absorbe ciertas entradas, estímulos o energías del medio circundante, y luego las procesa en su propia estructura, resultando a la salida un estado mas o menos acorde con los objetivos que había planteado. Otro concepto que no por ser tópico deja de ser importante, también analizable es el del mecanismo de retroalimentación, gracias al cual el resultado o producto es controlado y comparado con las finalidades, de modo que puedan realizarse los ajustes necesa- 
rios en las fases subsiguientes del funcionamiento del sistema y también puedan hacerse ajustes en las metas.

Ya hemos citado los componentes del sistema pero es importante saber, cómo están interconectados y cómo se conducen; dos componentes están interconectados cuando el producto, o salida de uno, sirve como entrada del otro. Los subcomponentes del sistema asumen ciertos valores o ideales que vienen determinados por los objetivos del sistema; cuando dichos objetivos realmente se alcanzan el sistema está en estado de equilibrio. Mediante la retroalimentación y las actividades reguladoras se puede acercar o alejar el sistema de ese estado de equilibrio determinado por los objetivos.

Se suele afirmar que el sistema está en equilibrio, cuando: a) su estado tiene una desviación mínima respecto del estado ideal que ha sido previamente definido por los objetivos del sistema (por ejemplo en el caso de objetivo de escolarización se ha logrado el $96 \%$ ), b) cuando, por alguna razón, el mecanismo de retroalimentación proporciona información insuficiente sobre la actividad y el estado del sistema respecto de sus finalidades (ejemplo, el órgano que elabora los presupuestos no ha recibido información sobre el proceso educativo y sigue asignando los mismos fondos año tras año), c) cuando los mecanismos reguladores, a pesar de la retroalimentación, son incapaces de lograr una nueva forma de acción (por ejemplo, se conocen las frecuentes incidencias negativas en la convivencia, en un Centro, y no se revisa su Reglamento interno).

En un sistema educativo han de tenerse presente las tareas básicas que se le han encomendado. Normalmente dichas tareas expresadas de un modo genérico, pueden darse por comunes en todo tipo de países. Se pueden concretar en las siguientes: 1) crear condiciones en que la educación del alumno se cumpla eficazmente; 2) ayudar a los alumnos a desarrollarse como individuos, de modo que sean capaces de participar activamente en la vida cívica y cultural, contribuyendo al ulterior desarrollo de la cultura y la sociedad; 3) transmitir la cultura desarrollada por las generaciones anteriores y compartir el desarrollo de la sociedad según determinados principios; 4) capacitar a los alumnos como agentes de la vida productiva que sirva para cubrir las necesidades presentes y futuras de la sociedad.

La actividad del inspector o supervisor cobra sentido cuando gracias a la información que por sí mismo ha recibido o la que se le transmite, respecto del funcionamiento del sistema permite apreciar diferencias entre el estado previsto para el sistema y su estado real. En ese sentido el inspector interviene en la toma de decisiones, es decir, en el proceso de resolver una situación problemática allí donde el problema estribe, en una discrepancia observada entre el estado previsto para el sistema y su estado real, implica, por tanto, la elección de una acción entre varias. El inspector, para los fines de toma de decisiones y credibilidad, desarrolla un papel fundamental, el de evaluador; el proceso de evaluación consiste en delinear, obtener y aplicar información descriptiva y estimati$v a$, sobre el mérito de algún objeto, tal como lo revela el diseño de su meta, la instrumentación y los resultados.

Por cuanto antecede entiendo que el inspector, cuya intervención es múltiple y le obliga a cumplir variados papeles, tiene su justificación en tres funciones: evaluación, toma de decisiones y asesoramiento. Consiste asesorar en dar orientación y consejo sobre algún campo o problema educativo. 


\section{La capacitación científica del inspector profesional}

Adoptado el criterio precedente sobre las funciones primordiales de la inspección, podemos sentar también algunos principios que configuren su profesión. Entiendo que el inspector ha de ser competente, discreto y prudente. La competencia comporta conocimiento teórico y dominio de técnicas; la discreción, capacidad de saber distinguir cuestiones y sus aspectos para saber determinar cómo, cuándo y a quién conviene; la prudencia permite la aplicación correcta de una norma o criterio en una circunstancia concreta.

El conocimiento teórico y las técnicas se insertan en lo que se suelen llamar competencias conceptuales y técnicas; la discreción y prudencia en las humanas.

Un recorrido por las áreas de conocimiento y disciplinas que cultivan los saberes específicos, nos puede permitir diseñar lo que pudiera ser un plan de estudios para el Inspector. He aquí mi propuesta:

Fundamentos teóricos de la educación.

Teoría general de sistemas.

Política de la educación.

Sociología de la educación.

Psicología de la educación.

Sistemas educativos comparados.

Fundamentos de administración.

Organización y planificación educativa.

Programación didáctica.

Modelos y técnicas de evaluación.

Teoría y técnicas de toma de decisiones.

Dinámica de grupos.

Métodos de investigación y estadística aplicada a la educación.

Evidentemente la capacitación en las materias precedentes es compatible con una experiencia docente que, a su vez, comporta la existencia en el Inspector de otra faceta, hasta ahora adoptada normalmente como elemento fundamental de su capacitación, a saber, pertenecer al profesorado en alguno de los niveles en que se configura el sistema educativo; dicha condición implica el haber sido formado en una especialidad concreta de la enseñanza.

\section{Alternativas académicas para la formación y titulación del Inspector}

Evidentemente, en congruencia con la postura adoptada en cuanto queda dicho, entiendo que la preparación científica del Inspector corresponde a la Universidad, en tanto que institución superior de docencia e investigación.

Los diversos Departamentos universitarios que componen la Universidad cuentan con los especialistas y los medios para cumplir este cometido. Esta proyección es la deseable no sólo para España, sino para todos los países del entorno europeo. 
Si se trata de encuadrar los estudios en los ciclos en que se divide actualmente nuestra enseñanza universitaria, evidentemente debe hacerse en el segundo ciclo o licenciatura, o bien en los estudios de postgrado. Podría quedar encomendado a un nuevo Instituto Universitario, que pudiera llamarse de Supervisión y Planificación educativas. Otra alternativa, también prevista en el ordenamiento jurídico de la Universidad, concretamente en el artículo 28 de la Ley de Reforma Universitaria, que en su punto tres dice: «las Universidades en uso de autonomía podrán impartir enseñanzas conducentes a la obtención de otros diplomas y títulos»; en la vigente normativa se desarrolla con más minuciosidad y precisión la referida posibilidad de titulación específica para los Inspectores, si nos atenemos a los Decretos reguladores de estudios de tercer ciclo y otros estudios para postgraduados (R.D. 185/1985 de 23 de enero), y al de obtención, expedición y homologación de títulos universitarios (R.D. 1496/1987, de 6 de noviembre).

En el orden jurídico se debe hacer constar que la Universidad puede expedir dos clases de títulos, aquellos que tienen carácter oficial y validez en todo el territorio nacional y los llamados Diplomas y títulos propios de las Universidades. Solamente los primeros surten efectos académicos plenos y habilitan para el ejercicio profesional, de acuerdo con la normativa vigente. Con lo expuesto es evidente que la aspiración debe tender a que la titulación de Inspector alcance la plenitud de reconocimiento.

Otra alusión pertinente es la que se refiere al perfeccionamiento de los inspectores, imprescindible como lo es para cualquier profesional. Aquí se puede contemplar la doble alternativa de ser encomendado a una institución oficial o bien la realicen las asociaciones profesionales, dotadas de los medios y subvenciones pertinentes.

Cualquier procedimiento que a estos fines se adopte lleva consigo establecer vías permanentes de comunicación entre distintos organismos, y a su vez esta comunicación sea comprensiva tanto de diversos entornos territoriales dentro de España cuanto de países que tienen afinidad intereuropea. No solamente reuniones periódicas de contacto y discusión como pueden ser Seminarios, Jornadas o Simposios, sino la publicación de revistas o al menos una revista profesional, donde se recojan investigaciones, estudios, noticias, documentación legislativa y todo tipo de información de interés general. Lo ideal sería que los sindicatos o asociaciones profesionales se integrasen en una federación europea y colaborasen en la publicación de una revista europea de inspección educativa.

En cualquier procedimiento de perfeccionamiento y actualización que se pueda establecer ha de mantenerse el criterio de que Universidad, administraciones educativas y asociaciones profesionales deben ser habituales partícipes, dado que la finalidad perseguida es la mejor capacitación de los profesionales de la inspección para prestar el servicio de la educación. La separación y mutua ignorancia de dichos sectores propicia carencia de rigor formativo, ineficacia y posiblemente solapamientos y campos de indefinición.

Es muy conveniente asumir, para los fines de formación y perfeccionamiento de los inspectores, las conclusiones adoptadas en el «II Simposio europeo de Inspección Educativa» que fueron recogidas bajo el epígrafe: «Perspectivas de la Inspección Europea. Proyectos europeos para inspectores de educación. Canales de comunicación». Intercambio de inspectores entre diversos países europeos, mediante stages que respondan a un programa previo de mutuos planteamientos sobre formación y desarrollo de técnicas e instrumentos específicos de la función inspectora. 


\section{SISTEMA EDUCATIVO E INSPECCIIÓN}

\section{Administración educativa e inspección}

Sin entrar en las posibles alternativas que para el sistema educativo se derivan de las teorías sobre Administración y Organización, cuyos representantes figuran en la historia de esta ciencia (Taylor, Fayol, Gulick, Urwick, Max Weber, Elton Mayo, Odierne y otros muchos), optamos tal como lo venimos haciendo a lo largo de esta exposición, por adherirnos a la Teoría General de Sistemas.

En un Estado de derecho el sistema educativo funciona como un subsistema de la organización social; administrativamente se establece, normalmente, como un departamento gubernamental cuyo fin es alcanzar las metas educativas que el poder ejecutivo determina. En muchos sentidos puede ser comparada la educación con un gran campo de cultivo en el que, un esfuerzo a largo plazo, gracias al esfuerzo y la credibilidad, puede asegurar la calidad del producto. Dicho gran campo es alimentado por las oportunidades que proporcionan los recursos físicos, técnicos y económicos, que pueden reunirse para contribuir a su crecimiento y obtener un producto que puede asumir varios valores.

Lo que ahora pretendemos considerar es como, organizativamente, aparece el sistema de la educación en un contexto que le da un contenido práctico determinado. Recordemos que en el seno de los sistemas hemos reconocido distintos niveles que suponen jerarquización, y en los cuales se pueden asumir papeles con distinta responsabilidad. En el nivel superior la filosofía básica y estructuradora de la educación atiende a circunstancias ambientales de carácter general y a las aspiraciones de la sociedad en armonía con otros sectores de la política, como puede ser lo social, económico, sanitario, seguridad, lo laboral, etc. Estos principios constituyen un punto de partida ideológico; dicho sustrato es la base de la planificación, en la cual aparece la necesidad de optar, por tanto de tomar decisiones, lo que comporta prioridades y selección. Estas decisiones son generales y desarrollan funciones directrices para todo el sistema. Quienes desarrollan la tarea en ese nivel superior son responsables de la política que se traza y del planeamiento e instrumentación de esa política; como en ese nivel se trata de dirigir el sistema hacia metas, en lo cuantitativo y en lo cualitativo, las especifican los políticos de la educación.

En el nivel segundo del sistema, se encuentran funciones vinculadas a los recursos y requieren toma de decisiones sobre estructuración, dado que existen medios alternativos para alcanzar las metas; las estructuras operativas se establecen para acciones detalladas, basadas en la plena comprensión de los objetivos, recursos y límites del sistema. Se encuentran en este nivel los funcionarios superiores cuya responsabilidad es abitrar recursos normativos, profesionales, de personal y financieros.

El tercer nivel incluye decisiones de programación mas concretas y específicas. Se especifican y revisan normas y reglamentaciones. En lo profesional se desarrollan actividades curriculares y de formación de personal docente; se facilita también la asignación de recursos para las distintas necesidades, tales como edificios, equipos, apoyos materiales, etc. Todo ésto requiere personal especializado, profesionales, administrativos y de planeamiento. La participación de los inspectores, en este nivel, es imprescindible en el campo de lo profesional, lo legal y del personal. Los Inspectores pueden aportar principios, criterios y técnicas, prácticas rutinarias y transmitir información en forma de observaciones, informes y juicios. 
El tercer nivel se refiere a la puesta en práctica o instrumentación de programas y entornos ambientales. En él se desarrollan funciones de liderazgo y comunicación; los inspectores pueden contribuir a la distribución de tareas de acuerdo con la especialización e intereses personales; algunos serán más eficientes para los aspectos administrativos y directivos, otros para la tarea pedagógica, para la actualización profesional o para intervenir en decisiones precisas de gasto y contabilidad.

El cuarto y último nivel citado, que en el orden de prioridad está más cerca del campo operativo de la enseñanza, se localiza la función de evaluación de las observaciones, experiencias e interpretaciones que resultan del nivel anterior, es decir, del proceso de instrumentación, para corregir tanto propósitos cuanto prácticas. Estamos en el momento de la retroalimentación, de la corrección y reasignación de recursos, lo que supone el comienzo, la vuelta a empezar de nuevo, de todo el proceso.

La inspección puede considerarse como un subsistema dentro del sistema educativo y tiene una estructura cuyas raíces están en los procesos a nivel individual, y la cúspide en los problemas de recursos a nivel de estructuración, pero también con responsabilidades intermedias. También puede la inspección estar inserta en el nivel de planificación en función asesora. De cualquier forma los niveles mencionados del sistema en que se estructura la educación, pueden identificarse con los escalones promocionales que deberían estar abiertos profesionalmente a los inspectores.

El hecho de que los inspectores tengan tareas definidas en cada uno de los niveles del sistema hace muy compleja su acción e impone un análisis a fondo de los distintos papeles que pueden asumir. Aunque los inspectores a título individual, rara vez toman decisiones que integran el proceso educativo general, desempeñan una importante función como agentes de toma decisiones, ya que aportan observaciones, interpretaciones, juicios y experiencias a las instituciones, organismos e individuos que realmente deciden y tienen que asumir las consecuencias. La inspección presta una gran contribución a las tareas de preparar, comunicar, instrumentar, conducir y sobre todo de coordinar la empresa educativa.

\section{Principales modalidades en los papeles de la Inspección}

En toda organización sus participantes desempeñan papeles y precisamente el sistema de papeles organizativos proporciona estabilidad a aquélla. El participante en una organización aporta recursos básicos propios, es decir, sus capacidades tanto físicas como cognitivas y sociales. Además debe tenerse en cuenta que por parte del participante existen necesidades que intenta satisfacer y precisamente algunas de ellas las quiere ver satisfechas a través del papel que asume.

Los recursos que la organización reclama se definen por los papeles. Las tareas específicas son las acciones que la organización espera de quienes desempeñan los papeles.

Analizada la estructura de la organización administrativa de la educación y vistos los niveles en que los inspectores pueden actuar podemos hablar de papeles institucionales; papeles que llamamos «adquiridos» dado que requieren las capacidades y cualidades de que hemos hablado (para distinguirlos de otro tipo de papeles, por ejemplo, los biológicos afectos a todas las personas de modo natural). 
En una organización la existencia de una estructura de metas supone una conducta destinada a alcanzarlas, independientemente de que existan otras metas (valores, normas, propósitos) y sanciones que originan premisas y límites dentro de los cuales se deben desempeñar las actividades. Los agrupamientos particulares de actividades pueden considerarse como programas que sirven para especificar las conductas requeridas demandadas por la organización y que están, por lo tanto, vinculados con su estructura de metas; el programa incluye también una descripción de la situación que evoca. Llegados a este punto podemos decir que, el papel es el conjunto del programa asociado con la conducta de un individuo aislado.

Otra cuestión a analizar es la jerarquía, es decir, una meta situada en el centro indica que lo es para un nivel inferior, a su vez ella misma es un medio para otra de nivel superior. Esto supone que los programas, para alcanzar esas metas, tienen también una forma jerárquica; lo que a su vez indica una jerarquía en los papeles.

Una serie de programas de la organización requieren la supervisión de otras personas, es decir, la autoridad formal sobre otros individuos de la organización. Cuando la supervisión se ejerce sobre la instrumentación o ejecución del programa por parte de otros, estamos ante una función gerencial. El proceso de supervisión implica trabajar con y mediante individuos y grupos para alcanzar metas. La autoridad formal permite al supervisor ordenar el cumplimiento de ciertos papeles u obligaciones institucionales. He aquí una de las funciones que puede adoptar distintos papeles; suelen ser, planificar, organizar, dirigir y controlar.

Recordemos ahora que un momento clave en un sistema es el de la comprobación de hasta que punto su sentido operativo y resultados se acercan o no a las metas, momento en que dicha retroalimentación indica la necesidad de tomar decisiones. Precisamente los inspectores intervienen en dicha función global de toma de decisiones, la cual se desarrolla mediante el desempeño de los papeles siguientes: evaluación, comunicación, programación y liderazgo.

Pasaremos ahora a analizar las variaciones que se aprecian cuando se asumen los papeles anteriormente citados según las funciones desempeñadas y el modo de realizarlas.

Variantes en la representación de los papeles de acuerdo con las funciones y modos de actuación

Recordando, de nuevo, que el papel es el conjunto del programa cuando se asocia con la conducta del individuo que lo representa, cabe brevemente aludir a papeles desempeñados para el desarrollo de la función global de supervisión.

El planificador ha de establecer metas y objetivos, y desarrollar programas de trabajo, mostrando cómo se puede proceder para alcanzar dichas metas y objetivos.

El organizador ha de integrar los recursos de forma programada para que incidan conjunta y ordenadamente personas, programas y materiales (habitualmente el término que se emplea en nuestra cultura es el de coordinador).

El controlador ha de retroalimentar los resultados y hacer el seguimiento para comparar los logros con los planes y hacer ajustes cuando sea necesario.

El director ha de comunicarse con las personas, motivarlas y demostrar seguridad. Su principal cometido es asegurar el empleo activo de los recursos tanto humanos como materiales, para que los efectos a corto y largo plazo sean favorables. 
Considerando ahora la otra función capital, anteriormente citada, cual es la toma de decisiones, vamos a distinguir los papeles de evaluador, comunicador y líder.

El evaluador presta su apoyo para la instrumentación curricular, intentando, por una parte detectar el grado de concordancia existente entre las intenciones (objetivos) y las observaciones y, por otra, buscar pruebas sobre la importancia de algunos requisitos previos y procedimientos para el aprendizaje; necesita dominio de técnicas para orientar la conducción de los recursos y de la acción.

El comunicador suele conjugar tres aspectos, el administrativo, el profesional y el personal. Lo administrativo en la comunicación tiene como objetivo lograr un flujo de información eficiente, sin obstáculos y mediante canales bien establecidos, empleando un código formal que sintetiza y da precisión y claridad a los mensajes. El modo de comunicación suele ser vertical. En el caso de comunicación profesional su objetivo es difundir una amplia información para aumentar la capacidad de resolver problemas; se da interactividad, se tiende a conjuntar esfuerzos; el modo de comunicación es horizontal. El otro papel es el de comunicador personal; se da una comunicación afectiva más que de transmisión de contenidos, puede darse incluso en un entorno formal, pero siempre tiene carácter privado y confidencial.

El papel de líder suele presentarse en tres acepciones. En la primera que consideramos orientamos la actividad hacia la tarea, la cual supone en quien representa el papel un liderazgo indirecto en el sentido de que se apoya en el poder que el cargo le otorga, y desempeña sobre todo tareas técnicas, gran cantidad de su esfuerzo lo ejerce mediante control externo; el modo de conducta es estrictamente jerárquico. Si se trata del líder orientado a la efectividad, ejerce el planeamiento, la organización, la dirección y el control de la estructura del trabajo; además de mostrar elevado interés para las metas y tareas instrumentales se preocupa de observar cuáles son los intereses, aptitudes y bienestar de los individuos implicados. Se trata de una actitud mixta y es oportuna cuando se trabaja con personas competentes y bien experimentadas que a cambio de autonomía y confianza para las tareas rutinarias asumen el compromiso para lo nuevo o lo discrecional. En último lugar el líder puede estar orientado hacia las relaciones, para quien el bienestar de cada uno de los participantes adquiere suma relevancia, es un estilo centrado en la persona; prevalecen las relaciones interpersonales; es un miembro del grupo que se une a sus subordinados, es como uno de ellos; la atmósfera de trabajo es amistosa y permisiva.

\section{Metas y fines operativos para la Inspección: niveles y entornos de toma de decisiones}

Retomamos la definición dada sobre toma de decisiones en el contexto de la teoría de sistemas, a saber, el proceso de resolver una situación problemática allí donde el problema estribe en una discrepancia observada entre el estado previsto para el sistema y su estado real. Los pasos típicos son: las fases de orientación, clarificación, instrumentación y conducción.

En la orientación es necesario establecer los criterios que sirvan de base para la aceptación o el rechazo de la situación a que se accede; es precisa la definición del estado final deseable, de los requisitos mínimos y de las metas específicas, analizar los elementos, sus relaciones y las deficiencias de la estructura o función; después generar y 
establecer alternativas, apreciando los aspectos positivos y negativos de cada una de ellas.

En la fase de clarificación, se elige una de las alternativas, mediante razonamientos explícitos y justificaciones concretas; tomada la decisión hay que hacer una asignación real de recursos. Sigue la fase de instrumentación que lo es de aplicación práctica; es un momento complejo porque han de interactuar personas, recursos y métodos. En la fase de conducción hay que hacer el seguimiento que consiste fundamentalmente en ajustes menores y revisiones de interpretación. A esta misma fase corresponden la evaluación y el reajuste. El evaluar supone, teniendo en cuenta finalidades y objetivos, determinar el significado de la información observada. Tanto los efectos cuanto las ventajas y problemas de la decisión se evalúan teniendo en cuenta entradas, procesos y productos de la decisión original. En último lugar se hacen los reajustes para aproximarse lo más posible a las finalidades originales.

Los niveles de toma decisión son resultado de la propia estructura del sistema educativo, puesto que la educación se desarrolla en diversos niveles de la administración, razón por la que se habla de decisiones a nivel político, de programa y de enseñanza. En el primero de los niveles se realiza la planificación, se señalan las metas como finalidades generales, de acuerdo con el entorno económico y sociocultural, para el desarrollo de la sociedad. Los problemas que surgen a este nivel se refieren a juicios de valor, a lo deseable y a los recursos disponibles. Los objetivos, en este nivel, se definen en términos generales, pues se relacionan con tendencias y metas a largo plazo. La evaluación de los efectos de las decisiones a este nivel político, consisten en un control general de la calidad del trabajo y de los productos del sistema. En el campo de las decisiones a nivel de programa (programas educacionales, directrices y materiales de enseñanza), se trata de producir y programar el empleo de los recursos asignados a los objetivos generales. Los problemas que surgen son tanto de orden cuantitativo como cualitativo. Los objetivos se definen en términos bastante específicos; la evaluación de la instrumentación de decisiones queda referida a la llamada evaluación del currículum en que se vinculan, metas educativas, aprendizaje del alumno y factores ambientales; el fundamental criterio de juicio es aquí el aprendizaje del alumno. El tercer y último nivel es el de enseñanza, se trata de decisiones que tienen relación con el alumno y forman parte del proceso general de interacción entre profesor, alumno, materiales de enseñanza y métodos utilizados. Aquí es donde se dan las definiciones más precisas de los objetivos del aprendizaje; los problemas se vinculan al diferente ritmo de aprendizaje; la toma de decisión se da en el trabajo cotidiano. La evaluación suele servir para dos funciones; como retroalimentación para dar al alumno y al profesor una guía en sus actividades, o bien para indicar una escala u orden de mérito que se empleará con fines pronósticos y de calificación.

Tomamos de Stufflebeam (1976) el diseño de entornos de decisión, que brevemente aludimos.

Al canalizar las dimensiones básicas que organizan los entornos de decisión se pueden considerar dos dimensiones: la del cambio educacional que puede ser pequeño o grande, y la del dominio de la información, para apoyar el cambio que puede ser alto o pequeño.

La primera de las dimensiones citadas mide la importancia de la decisión, en la medida en que, por una parte, se desvía de las prácticas que más predominan y, por otra, según la amplitud del campo aunque se extienden las consecuencias de la decisión. En la 
segunda dimensión se considera la existencia de información y la capacidad del cliente para utilizarla en su forma actual. Al considerar simultáneamente las dos dimensiones citadas se ponen de manifiesto cuatro entornos de decisión diferentes. Resultarán la toma de decisiones homeostática, incremental, neomovilista y metamórfica, respectivamente.

El primer tipo de toma de decisión, llamado homeostático, se realiza para introducir en el sistema pequeños cambios y se apoya en un alto dominio de la información. Recordemos que la homeostasis es un concepto biológico y se trata de un conjunto de fenómenos de autorregulación que conducen al mantenimiento de una relativa constancia de las propiedades de ciertos sistemas influidos por agentes externos. Se trata de una actividad restauradora destinada a mantener el equilibrio normal en un sistema educativo; se guía por normas técnicas y por un sistema rutinario de toma de información. Entornos de decisión de este tipo se aprecian en todos los niveles educativos; desde la planificación y establecimiento de metas educativas, propio del sector de la política educativa, cuando, por ejemplo, tratan de mantener una cierta proporción docente/discentes, al nivel en que los docentes y encargados de diseñar los programas de las materias intentan enseñar los instrumentos culturales con métodos y materiales tradicionales.

Un segundo tipo de entorno de decisión es el incremental; se trata en él de un desarrollo para cambios pequeños; el dominio de información que se requiere es bajo. Es la tendencia a un esfuerzo contínuo para introducir mejoras. Lo que quieren hacer es mejoras pequeñas y seriadas, desplazando el programa en su conjunto a un nuevo equilibrio. A diferencia de las decisiones de tipo homeostático que tienden a corregir el programa y devolverlo a su estado anterior. Otra diferencia es que mientras las decisiones homeostáticas se apoyan en normas técnicas y en una continua información de rutina, las evaluaciones en el caso de incrementalismo son específicas y se apoyan en una escasa asistencia de información. La mayor parte de las llamadas innovaciones educativas son de este tipo incremental por tratar de intentos por mejorar los programas vigentes sin correr el riesgo de un grave fracaso. Estos cambios se apoyan en el ensayo y el error y exigen la asignación de recursos especiales.

Pasamos ahora al análisis de decisiones para conseguir cambios comparativamente grandes. Se trata de actividades innovadoras para inventar, probar y difundir nuevas soluciones a problemas significativos. Este cambio denominado neomovilista, suele aprovechar alguna oportunidad de desarrollo tecnológico (por ejemplo la informatización) y otras veces una determinada situación crítica de la sociedad. La evaluación que se emplea suele adoptar fórmulas de investigación dispuestas de modo específico. Al principio se adoptan técnicas de exploración para el esfuerzo del cambio, verdaderos estudios que incrementan su rigor a medida que se avanza en el cambio. Este tipo de decisión es el que requiere mayor inversión en evaluación. La evaluación ha de ir acompañada de una organización efectiva para que la retroalimentación se comunique fluidamente y se haga notar en nuevas asignaciones de recursos.

Finalmente nos referimos al tipo de toma de decisiones que implica grandes cambios, llamada metamórfica. Se quieren producir cambios completos en un sistema educativo. Ha de basarse en el riguroso conocimiento del modo de hacer los cambios. Para ella se necesita un alto dominio de la información y por tanto el establecimiento de redes fluidas y con alta coordinación. También ha de apoyarse en una buena evaluación. Hay que establecer fuertes bases de conceptualización; es decir, todos los intervinientes en el 
proceso deben ser aptos para comprender los fundamentos del cambio, su necesidad y estar de acuerdo con los objetivos de la organización en su totalidad y no sólo sobre la base de las metas y necesidades del propio grupo que le es lo más inmediato.

\section{ESTATUTO DE LA INSPECCIÓN EDUCATIVA EN ESPAÑA}

\section{Hitos más recientes}

La inspección educativa en España ha tenido una reglamentación, en sus orígenes y hasta tiempos recientes, exclusivamente para el nivel obligatorio de enseñanza (se inicia en 1849). Aproximándonos cronológicamente la inspección llamada de bachillerato aparece en 1938 y la entonces llamada coordinación de formación profesional en 1975. Para la enseñanza primaria la norma de mayor desarrollo aparece en 1967. Por Decreto de 1973, en desarrollo del articulado correspondiente de la Ley General de Educación de 1970, se unifica en un servicio único la función inspectora, afectando a los niveles primario y secundario de la enseñanza. Prescindiendo del análisis de los precedentes citados entraremos en la consideración de la normativa vigente a cuyo fin procede tener en cuenta tanto las disposiciones de la administración central cuanto de la autonómica en España. Si bien es comprensivo que en una actitud maximalista se tuvieran en cuenta también los reglamentos de los países europeos, fundamentalmente los que integran la Comunidad Europea, hemos diferido tal planteamiento para nueva ocasión.

El análisis que vamos a hacer (presentado simultáneamente en cuadro sinóptico) se diseña en tres dimensiones: funciones a cumplir, papeles a representar y capacidades requeridas.

Este estudio es congruente con el resto de los aspectos presentados en esta exposición. Por una parte servirá para apreciar hasta qué punto la realidad constatada sobre la inspección en España se corresponde con el estudio formal presentado a través de la teoría de sistemas, por otra para considerar si la configuración, funciones, competencias y papeles que a la inspección se le atribuyen pueden satisfacer las necesidades que presenta un proceso de innovación, cual es el diseñado en la vigente Ley de ordenación general del sistema educativo, o en su caso, estudiar los cambios que en ella haya que introducir para que, conservando su carácter profesional, contribuya eficazmente a la innovación educativa.

\section{Normativa que emana de la Administración Central del Estado}

Nos referimos aquí al R.D. 1524/1989 de 15 de Diciembre que regula las funciones y la organización del Servicio de Inspección así como el acceso a los llamados puestos de trabajo de la función inspectora, y por otra parte a la Ley de ordenación general del sistema educativo de 3 de Octubre 1990 en su Título cuarto, artículo 61.

El Decreto de referencia alude sucesivamente a: funciones y atribuciones de la inspección, organización y funcionamiento, procedimiento de acceso, concursos de traslados y provisión de puestos de nivel central y jefatura provincial. Hace una salvedad en la estructura específica de la Inspección Provincial de Madrid. 
Por lo que a funciones se refiere, cuestión más importante para los fines concretos de esta exposición, enumera las de: asesorar (a los Centros), colaborar (con unidades de planificación, sólo a nivel provincial; en reformas educativas, perfeccionamiento del profesorado, renovación pedagógica, programas experimentales por lo que se refiere a su difusión, seguimiento y evaluación); evaluar (el rendimiento educativo de los Centros docentes y servicios y la ejecución y desarrollo de programas y actividades promovidos o autorizados por el Ministerio de E. y C.); informar (sobre todo aquéllo para lo que se le requiera por la autoridad o haya conocido en el ejercicio de su actividad); velar (por el cumplimiento de la normativa de educación en Centros, servicios y en la ejecución y desarrollo de programas y actividades de tipo educativo).

Las funciones que se acaban de citar se ejercen en el campo siguiente: Centros, programas, actividades y servicios del sistema educativo, excluida la Universidad. En cuanto al modo de actuación, especificado bajo el epígrafe de atribuciones, se concreta en: visitar Centros, servicios e instalaciones tipo educativo promovidas o autorizadas por el Ministerio de E. y C. y accediendo a la documentación académica y administrativa de centros y servicios educativos. Para el ejercicio de su función son considerados autoridad pública. La visita se concluye en informe, como sistema habitual de trabajo.

El sistema de organización del servicio de inspección, es vertical, en el seno de la administración del Ministerio de E. y C.; a saber, Dirección General (de Coordinación y Alta Inspección), Servicio Central y Servicio Provincial; Demarcación territorial; Coordinador y equipo de inspectores en la base. Los inspectores a título individual no intervienen en toma de decisiones ni tampoco colegiadamente al no funcionar de acuerdo con el esquema de Consejo de inspectores.

El sistema de acceso es a partir de la condición profesional de docente. Las pruebas públicas han sido eliminadas y los méritos se conocen por constancia documental. Los perfiles se definen por niveles educativos y áreas disciplinares.

La otra norma a que nos referimos, posterior a la que acabamos de citar, puesto que data el 3 de Octubre de 1990, es la LOGSE. En su Título cuarto, referido a la calidad de la educación incluye a la Inspección educativa entre los siete factores de calidad que enumera.

En el artículo 61 queda establecido que «las Administraciones educativas ejercerán la función inspectora para garantizar el cumplimiento de las leyes y la mejora de la calidad del sistema educativo». Encomienda a la inspección educativa las siguientes funciones:

a) Asesorar e informar a los distintos sectores de la comunidad en el ejercicio de sus derechos y en el cumplimiento de sus obligaciones.

b) Colaborar en la mejora de la práctica docente y del funcionamiento de los Centros, así como en los procesos de renovación educativa.

c) Participar en la evaluación del sistema educativo.

d) Velar por el cumplimiento de las leyes, reglamentos y demás disposiciones generales en el ámbito del sistema educativo.

Para el desarrollo de dichas funciones se autoriza el acceso de los inspectores a centros, servicios e instalaciones en que se desarrollan actividades promovidas o autorizadas por las administraciones educativas.

Por lo que se refiere al esquema jerárquico, el nivel máximo se reserva al Estado al atribuirle la alta inspección para garantizar el cumplimiento de las obligaciones de los poderes públicos en materia de educación. 
Comparando el contenido de las dos normas aludidas resulta que mientras en esta última se especifican 5 funciones (asesorar, informar, colaborar, participar y velar), en la primera, es decir, en el R. Decreto 1525/1989, se constatan 6, al especificarse la de evaluar.

Normas reguladoras emanadas de las Administraciones autonómicas del Estado español

Para seguir el procedimiento adoptado en el punto anterior hemos consultado la normativa sobre inspección de la educación, emanada de entes autonómicos del Estado español a los que fueron transferidas las competencias en este sector. Se trata de las administraciones de Andalucía, Cataluña, Canarias, Galicia, País Vasco y Valencia.

En conjunto aparecen diferenciadas las once funciones siguientes: Asesorar, colaborar, controlar, coordinar, cumplir y hacer cumplir, estimular, evaluar, informar, participar, supervisar y velar. De ellas la más prolija en la asignación de funciones es la Autonomía de Andalucía, con ocho, y la menos el País Vasco que solamente enumera tres. En el orden cronológico todas las normas son anteriores a las dos normas provenientes de la Administración Central, por una parte, y de otra de las Cortes (con rango de Ley Orgánica).

Hay que hacer especial referencia a dos de las funciones que aparecen en la normativa que ahora comentamos y no hay constancia de ellas en las dos normas que hemos comentado en primer lugar. Nos referimos a la labor de coordinar los programas y servicios que se le encomienden (Galicia) y a la de supervisar la utilización de fondos públicos (Andalucía).

Otras notas diferenciales entre la normativa de los entes autonómicos son en el orden de estructura y funcionamiento, la referencia a la actuación diferenciada de los Inspectores en equipos, el de asesoramiento, y los subequipos de evaluación educativa y el de control administrativo; ambos establecidos por la Junta de Andalucía; consiguientemente con tal estructura, otra norma enumera las funciones comunes y las diferenciadas de cada uno de los equipos.

En congruencia con las funciones que se constatan en la normativa analizada hay que establecer los papeles que la inspección ha de desempeñar para un ejercicio profesional correcto. Partiendo del total de funciones descritas tomando en conjunto toda la normativa, es decir, analizando el máximo de funciones, se desprende que los inspectores tienen que asumir, según la función y circunstancias específicas los papeles siguientes: comunicador en las variantes de profesional, personal y administrativo; líder en sus variantes de orientación a la tarea, a la efectividad y a las relaciones humanas; agente de mantenimiento, desarrollo y guía; evaluador e intérprete de normas.

A su vez el ejercicio de los papeles citados, consecuencia de las funciones que tiene que asumir, traen como exigencia unas determinadas capacidades. Estas capacidades requeridas son la conceptual, técnica y humana. Estos tres ámbitos de capacidad identificados pueden sucintamente describirse así. Partiendo de la premisa de que la educación es un sistema, la capacidad conceptual supone la comprensión de las complejidades de la organización en su totalidad, por una parte, y por otra el área de organización a que se adapta la propia operación; de esta forma se puede actuar de acuerdo con los objetivos de la organización en su totalidad y no sólo sobre la base de las metas y necesidades del 
propio grupo inmediato. La capacidad técnica se entiende como aptitud para utilizar el conocimiento, los métodos, técnicas y equipos necesarios para el cumplimiento de tareas específicas adquiridas mediante educación, capacitación y experiencia. Finalmente lo que hemos llamado capacidad humana supone aptitud y juicio para trabajar con y mediante personas, comprensión de la motivación y aplicación del liderazgo.

Dicho ésto podríamos extender el estudio en el sentido de comprobar si es congruente la preparación que actualmente se exige para el acceso a la función inspectora con las capacidades y formación específica que vienen exigidas ya no por una definición formal de la profesión inspectora, sino por las funciones que la normativa del sistema educativo español ha desarrollado al respecto. Aparentemente creo que se ha tomado la parte por el todo, es decir, se ha extendido lo necesario al concepto de lo suficiente; quiero decir que la condición de profesional de la docencia no conlleva la formación específica del inspector, que según venimos considerando reviste una identidad profesional bien diferenciada.

\section{INNOVACIÓN EDUCATIVA E INSPECCIÓN}

\section{Innovación, toma de decisiones y evaluación}

Innovación, toma de decisiones y evaluación están estrechamente vinculados para los fines que en esta exposición pretendemos.

Genéricamente hablando, innovar es formular una nueva solución a algún o a algunos problemas cuya solución perseguimos. Aunque existen varios modelos para llevar a cabo una innovación, estimamos bueno para nuestras pretensiones el que sigue los pasos de investigar, desarrollar, difundir y adoptar. Es decir, plantear en primer lugar las bases del conocimiento que nos permitan formular la innovación; ordenar y sistematizar los componentes de la solución que proponemos; informar, después, para toma de conciencia entre los participantes en la innovación, avanzando más tarde hacia la demostración para crear un convencimiento; experimentar o poner a prueba el modelo, hacer congruentes las características de la innovación con el sistema educativo, o sea, implantarlo y finalmente establecerlo asimilando la innovación.

Indudablemente toda innovación requiere una serie de sucesivas tomas de decisión y las correspondientes evaluaciones.

Hemos considerado ya el grado o intensidad del cambio, al hablar de entornos de decisión, empezando en el nivel de homeostasis o mantenimiento del sistema y concluyendo en el metamórfico que exigía grandes cambios. Recordando también la estructura y funcionamiento de un sistema, hay en primer lugar un contexto (que hace referencia a los factores externos que no son sino componentes del sistema social del que el sistema educativo es un subsistema), la entrada (input), el proceso y el producto (out-put), todos estos momentos necesitan ser evaluados. Volviendo ahora a retomar la toma de decisiones es preciso distinguir tipos de decisión además de, simultáneamente, tener en cuenta los entornos (grados) ya citados.

Los tipos de decisión han de ser considerados necesariamente, atendiendo a los fines u objetivos o bien a los medios o instrumentos y, por otra, según se trate de actuar sobre 


\begin{tabular}{|c|c|c|c|c|c|c|c|c|c|c|}
\hline \multicolumn{8}{|c|}{ ÓRGANO DE PROCEDENCIA } & FUNCIONES & \multirow{2}{*}{\begin{tabular}{l}
\multicolumn{1}{c}{ PAPELES } \\
En los papeles destaca el \\
de intérprete de normas, \\
seguido por el de lider \\
para la tarea y la efectivi- \\
dad.
\end{tabular}} & \multirow{2}{*}{\begin{tabular}{|l} 
CAPACIDAD \\
$\begin{array}{l}\text { La capacidad } \\
\text { más requerida es } \\
\text { la conceptual, le } \\
\text { sitúe la social. }\end{array}$
\end{tabular}} \\
\hline 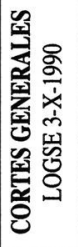 & 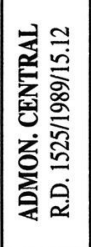 & 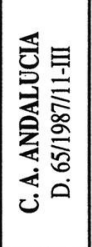 & 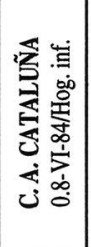 & 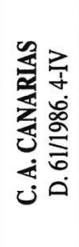 & 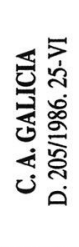 & 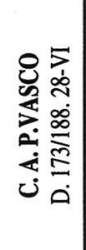 & 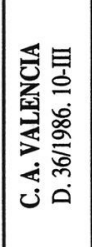 & $\begin{array}{l}\text { En el análisis de funciones que las normas atribuyen a la Inspección aparecen } \\
\text { once, si bien algunas pueden ser interpretadas como equivalentes. Aparecen con } \\
\text { mayor frecuencia: asesorar, evaluar, velar por el cumplimiento de normas e in- } \\
\text { formar. }\end{array}$ & & \\
\hline * & $*$ & * & * & $*$ & $*$ & $*$ & & $\begin{array}{l}\text { ASESORAR: - a los órganos unipersonales y colegiados de los Centros; - espe- } \\
\text { cialmente al profesorado; - sobre las competencias y atribuciones asignadas. }\end{array}$ & $\begin{array}{l}\text { COMUNICADOR } \\
\text { profesional } \\
\text { INTÉRPRETE }\end{array}$ & $\begin{array}{l}\text { CONCEPTUAL } \\
\text { TÉCNICA } \\
\text { SOCIAL }\end{array}$ \\
\hline \multirow[t]{5}{*}{ * } & $*$ & * & & * & & & & $\begin{array}{l}\text { COLABORAR: - en reforma educativa, perfeccionamiento profesorado, reno- } \\
\text { vación pedagógica, progr. experimentales, mejora prạ́ctica docente, funciona- } \\
\text { miento Centros, est. neces. educativas. }\end{array}$ & $\begin{array}{l}\text { AGENTE de manteni- } \\
\text { miento, desarrollo y guía. }\end{array}$ & CONCEPTUAL \\
\hline & & $*$ & & & * & $*$ & & $\begin{array}{l}\text { CONTROLAR: - el cumplimiento de la normativa legal en materia de educa- } \\
\text { ción en Centros públicos y privados. }\end{array}$ & $\begin{array}{l}\text { LIDER para la tarea, la } \\
\text { efectividad y relac. }\end{array}$ & $\begin{array}{l}\text { CONCEPTUAL } \\
\text { SOCIAL }\end{array}$ \\
\hline & & & & & * & & & COORDINAR: - los programas y servicios que se le encomienden. & $\begin{array}{l}\text { LIDER orientado a la } \\
\text { efectividad. }\end{array}$ & $\begin{array}{l}\text { CONCEPTUAL } \\
\text { SOCIAL }\end{array}$ \\
\hline & & $*$ & & & & & $*$ & $\begin{array}{l}\text { ESTIMULAR: a los integrantes de la comunidad educativa para una eficaz } \\
\text { participación y para conocimiento de derechos y deberes. }\end{array}$ & AGENTE de desarrollo. & $\begin{array}{l}\text { SOCIAL } \\
\text { TÉCNICO }\end{array}$ \\
\hline & $*$ & $*$ & & $*$ & * & $*$ & * & $\begin{array}{l}\text { EVALUAR: - el rendimiento educativo de los Centros, el rendimiento del sis- } \\
\text { tema, analizando organización, funcionamiento y resultados de Centros y servi- } \\
\text { cios; ej. y des. de programas y actividades. }\end{array}$ & $\begin{array}{l}\text { EVALUADOR } \\
\text { INTÉRPRETE }\end{array}$ & $\begin{array}{l}\text { CONCEPTUAL } \\
\text { TÉCNICA }\end{array}$ \\
\hline * & $*$ & $* *$ & * & $*$ & & & * & $\begin{array}{l}\text { INFORMAR, ATENDER Y DAR RESPUESTA: a los sectores de la comuni- } \\
\text { dad educativa sobre atribuc. y competencias, seg. normas; lo que requiera la aut. } \\
\text { competente; lo que conozca en el ejercicio de su actividad. }\end{array}$ & $\begin{array}{l}\text { PROFÉSIONAL, } \\
\text { PERSONAL, } \\
\text { ADMINISTRATIVO } \\
\end{array}$ & $\begin{array}{l}\text { TÉCNICA } \\
\text { SOCIAL }\end{array}$ \\
\hline \multirow[t]{2}{*}{ * } & & & & & & & & PARTICIPAR: - en la evaluación del sistema educativo. & $\begin{array}{l}\text { LIDER orientado a la ta- } \\
\text { rea. }\end{array}$ & $\begin{array}{l}\text { CONCEPTUAL } \\
\text { SOCIAL }\end{array}$ \\
\hline & & $*$ & & & & & & SUPERVISAR la utilización de fondos públicos. & INTÉRPRETE de normas. & $\begin{array}{l}\text { TÉCNICA } \\
\text { CONCEPTUAL }\end{array}$ \\
\hline * & $*$ & & * & $*$ & $*$ & & * & $\begin{array}{l}\text { VELAR, cuidar: - por el cumplimiento de leyes, reglamentos y demás disposi- } \\
\text { ciones educativas en Centros, programas, actividades y servicios. }\end{array}$ & INTÉRPRETE de normas. & $\begin{array}{l}\text { CONCEPTUAL } \\
\text { SOCIAL }\end{array}$ \\
\hline
\end{tabular}

ANÁLISIS DE FUNCIONES, PAPELES Y CAPACIDADES, REQUERIDAS PARA LA INSPECCIÓN EDUCATIVA EN ESPAÑA, según normas legales. A.B.G. 1992. 
las previsiones o sobre la realidad misma. Así resulta que, en este sentido, puede haber decisiones sobre planificación, estructuración, instrumentación y recuperación. Las decisiones de planeamiento se refieren a los fines de planeamiento de una política, es decir, a previsiones; son necesarias en todos los niveles de la jerarquía de toma de decisión pero, evidentemente, tienden a realizarse en el nivel de la política educativa. También tienen carácter previsorio, pero en este caso de procedimiento las llamadas de estructuración; se refieren a los medios para lograr las decisiones de planeamiento; al uso organizado de los recursos (humanos, materiales, de tiempo y espacio). Los grandes limitadores de este tipo de decisión son los presupuestos económicos y el tiempo. De un modo global estas decisiones proporcionan las operaciones necesarias para responder efectivamente a las decisiones políticas de planificación. En el mismo plano de los medios pero esta vez en situación real, se dan las decisiones de instrumentación; guian el uso real de los recursos programados; aunque en los niveles jerárquicos superiores este tipo de decisiones se den, lo son con poca frecuencia y de modo formal; realmente las decisiones de instrumentación ocurren en las instituciones donde se desarrollan las actividades cotidianas de la educación. Finalmente las decisiones de recuperación, también en el campo de la realidad, pero afectando a los fines, están relacionadas con la terminación o continuación de la acción. La principal fuente de esta toma de decisiones es la comparación entre los objetivos y los resultados. Precisamente en las estrategias formativas, por contraposición a las sumativas, se establecen frecuentes puntos de control a lo largo de toda la actividad, procurando hacer ajustes incrementales desde el comienzo del proceso de instrumentación.

Siguiendo al autor ya citado (Stufflebeam, 1976) la evaluación está especialmente indicada para los fines de toma de decisiones y de credibilidad, y se realiza trazando, obteniendo y aplicando información descriptiva y estimativa, sobre el mérito de algún objeto tomando como referencia su meta, instrumentación y resultados. La evaluación en lo social y por tanto en educación suele emplear criterios comparativos para no perder de vista decisiones anteriores y sus resultados para aprender de la experiencia; por otra parte la evaluación lleva consigo un registro de credibilidad de decisiones pasadas y de la información de que se disponía para tomar dichas decisiones. La evaluación en educación se aplica a un campo extenso, como puede ser el del aprendizaje de los alumnos, el personal, los programas, los proyectos, los materiales, instituciones, presupuestos, etc. De los distintos usos que se puede hacer de las actividades de organización de la educación nacen los tipos de evaluación. Poniendo la evaluación en relación con las cuatro clases de decisión a que anteriormente hemos hecho referencia resultan los tipos de evaluación del contexto, del input, del proceso y del producto.

La evaluación del contexto se pone al servicio de las decisiones de planeamiento, cuyo intento es definir, describir y estimar el contexto operatorio, es decir, el entorno físico, social y actitudinal en que se desarrolla la educación.Consiste en identificar necesidades, oportunidades y problemas, proporcionando las bases para determinar los objetivos que pueden ser adoptados como metas del sistema, ya sea en uno u otro nivel (nacional, territorial, centro, aula). Es una actividad continua y necesita de algún procedimiento de registro. La evaluación del «input» sirve para las decisiones de estructuración, lo cual permite determinar la cantidad y especificidad de los recursos que han de ser empleados para lograr los objetivos del proyecto. Hay que describir y valorar por ejemplo, los cursos (incluyendo, personal, tiempo, edificios, medios económicos, etc.) y 
la programación. No estudia pues las realidades sino las previsiones necesarias para la consecución de objetivos. En esta fase se suelen desarrollar proyectos piloto.

Pasando al campo no ya de lo previsible sino de lo real, nos encontramos con la evaluación del proceso y la del producto. En la evaluación del proceso se atiende al empleo real de los recursos disponibles para apoyar las decisiones de instrumentación durante el desarrollo de un programa. Sirve para proporcionar retroinformación a las personas que se encuentran comprometidas con las interacciones entre recursos, programas y beneficiarios del sistema. En educación el entorno a observar suele ser la institución educativa y el aula o grupo de alumnos. Este tipo de evaluación utiliza gran variedad de instrumentos y procedimientos para describir y juzgar el proceso, haciendo una retroalimentación inmediata tanto para el personal que interviene en el programa cuanto para el mantenimiento de registro de información. Finalmente nos referimos a la evaluación del producto cuyos objetivos se refieren a la descripción y estimación de los logros del programa, es decir a juzgar sobre la relación que existe entre el producto y las metas del proyecto; es la base para tomar decisiones de recuperación o reciclaje.

Es interesante añadir que la necesidad de aplicar los distintos tipos de evaluación que hemos descrito depende de la clase de decisiones y de la naturaleza del cambio buscado. Cuando se trata de seguir un programa que se está desarrollando la evaluación del contexto puede ser continua y hecha de modo sistemático; solamente cuando se ha tomado una decisión de planeamiento para realizar un cambio se deben aplicar los otros tres tipos de evaluación. Volvemos ahora al concepto de entorno del cambio para decir que cuanto mayor sea el cambio y más baja la información (el conocimiento que tienen los que lo han de decidir del cómo hacerlo), más amplia, formal y estructurada será la evaluación. Las evaluaciones de entrada, proceso y producto lo son en gran medida necesarias cuando el cambio es neomovilista, pero apenas se desarrollan cuando el entorno de la decisión es homeostático o incremental.

\section{La innovación que propugna la vigente política educativa en España}

Cuanto hemos venido exponiendo queda justificado como precedente de una pretensión: analizar las categorías, cantidad y tipo de cambio que se configura para el sistema educativo español y su relación con las funciones, papeles y capacidades del inspector.

El texto de la LOGSE cuando se refiere a investigación y elaboración de proyectos educativos (art. 59.1) cita innovaciones curriculares, metodológicas, tecnológicas, didácticas y de organización de los Centros. Aunque dichas innovaciones, tal como nominalmente se expresan, no sean fáciles de discernimiento, dado que se pueden incluir y excluir entre sí, dan a conocer líneas generales.

El texto normativo aludido, a mi entender, quiere poner énfasis en tres grandes aspectos. El cualitativo, en que hace especificaciones importantes tales como: desarrollo de la orientación educativa e intervención psicopedagógica, evaluación del sistema educativo y apoyo al profesorado en el desarrollo del currículo. En lo estructural: la educación básica a la que da carácter comprensivo, ampliación de la educación básica, diferenciación entre educación primaria y secundaria, conversión de lo preescolar en una etapa dentro del sistema educativo y la mayor relevancia e inclusión de la formación profesional. En cuanto a ordenación de las enseñanzas: evaluación interna del aprendi- 
INTERRELACIONES ENTRE SISTEMA EDUCATIVO, TOMA DE DECISIONES, INNOVACIÓN Y EVALUACIÓN

A.B.G. 1992

NIVEL DE SISTEMA

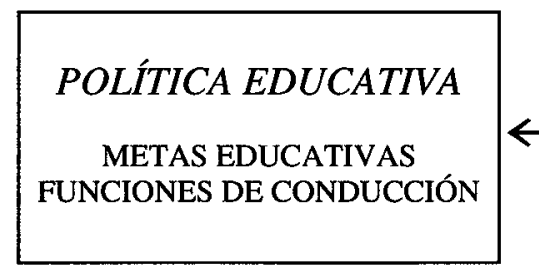

TOMA DE DECISIÓN

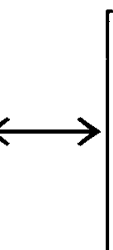

PLANEAMIENTO

DECISIONES SOBRE METAS SELECCIÓN DE PRIORIDADES
INNOVACIÓN

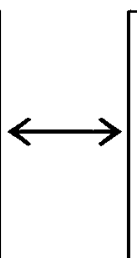

\section{INNOVACIÓN}

\section{INVESTIGACIÓN}

DETERMINACIÓN DE BASES DE CONOCIMIENTO PARA FORMULAR LA INNOVACIÓN
EVALUACIÓN

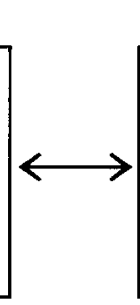

\section{EVALUACION}

\section{CONTEXTO}

DESCRIBIR Y ESTIMAR EL

DESCRIBIR Y ESTIMAR EL
ENTORNO FÍSICO, SOCIAL Y ENTORNO FISICO, SOCIAL Y
ACTITUDINAL DE LA EDUCAACTITUDINAL DE
CIÓN
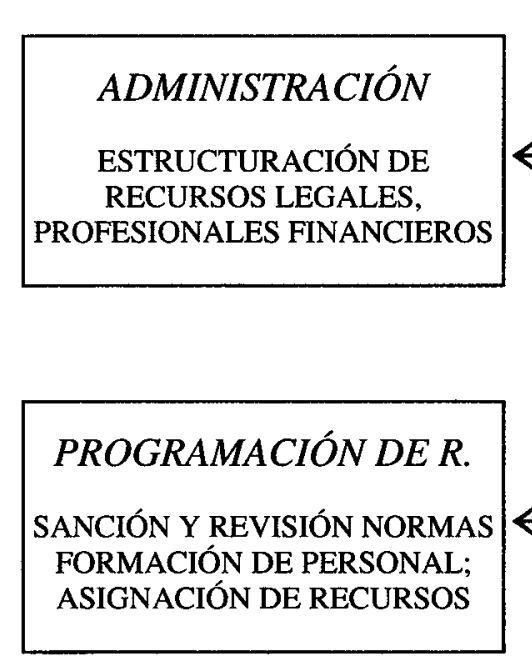

FORMACIÓN DE PERSONAL ASIGNACIÓN DE RECURSOS

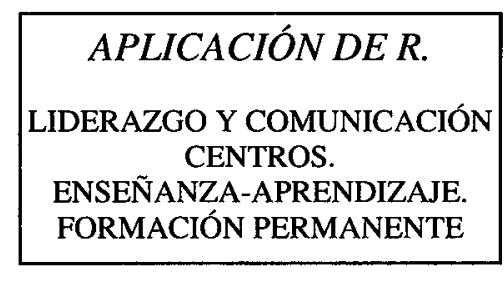

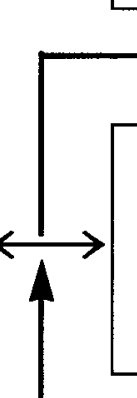
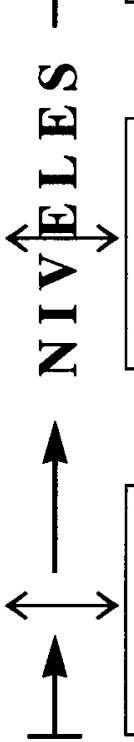

$\uparrow$

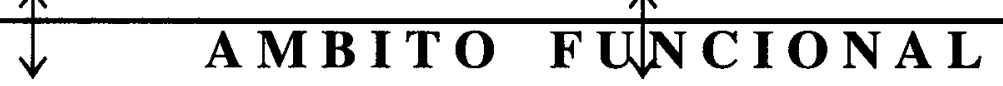

\section{ESTRUCTURACIÓN \\ DECISIONES SOBRE} ESTRUCTURAS OPERATIVAS Y MEDIOS
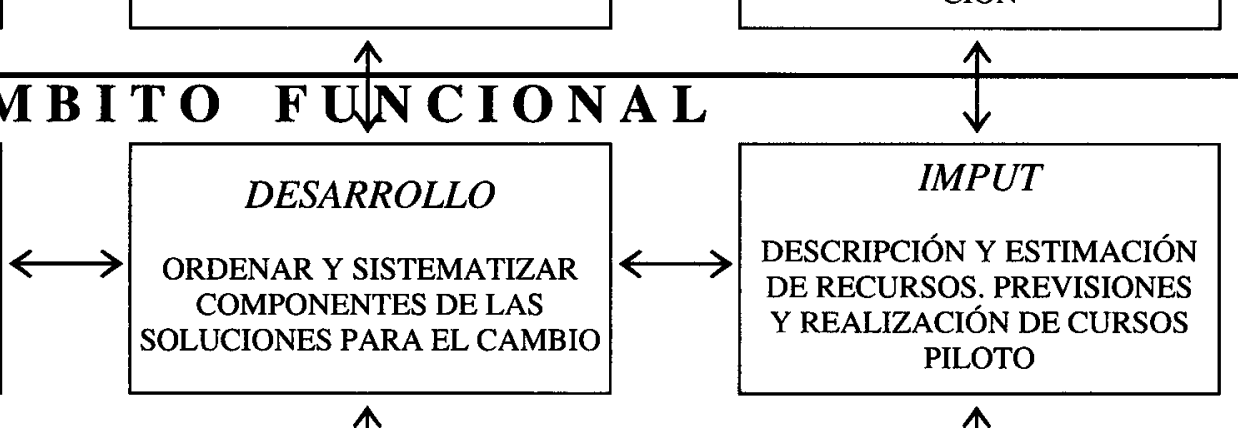

DESCRIPCIÓN Y ESTIMACIÓN DE RECURSOS. PREVISIONES Y REALIZACIÓN DE CURSOS PILOTO
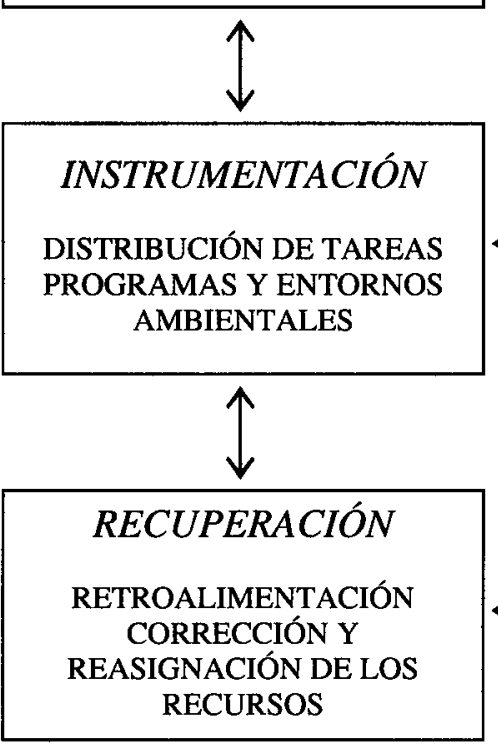

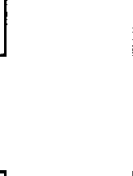

\section{DIFUSIÓN}

DEMOSTRAR: INFORMANDO Y CONVENCIENDO EXPERIMENTAR

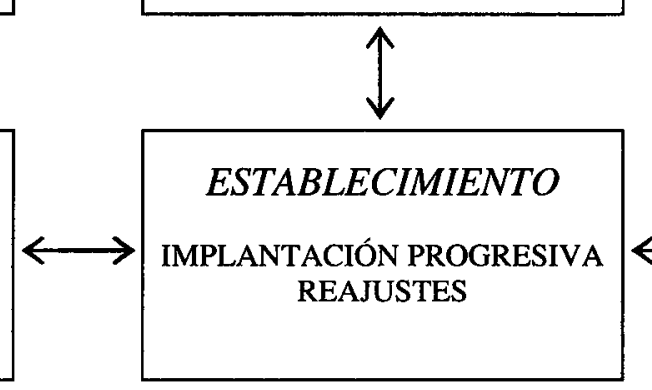

ACTUA EN TIEMPO REAL

PROPORCIONA

RETROINFORMACIÓN SOBRE PROGRAMAS Y RECURSOS

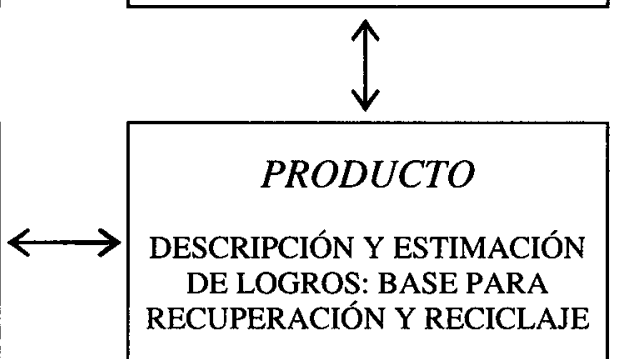


zaje por parte de los centros (aspecto que también puede incluirse en criterio cualitativo), nueva estructura de ciclos y áreas y la especial elaboración del currículo.

La búsqueda de nuevos matices para determinar con mas precisión la innovación propugnada nos lleva a apreciar determinados tipos de cambio. Por ejemplo se percibe necesidad de alteración de las estructuras en virtud de haberle dado un carácter comprensivo a la educación básica, con la introducción de la educación secundaria obligatoria, el acortamiento de la etapa de bachillerato, alteraciones que conllevan la sustitución parcial del profesorado. Otro tipo de cambio que viene exigido es la reestructuración del currículo, de los Centros y subsiguientemente de los planes de estudio del profesorado; se producen adiciones en lo cualitativo según hemos indicado al enumerar tres aspectos en la innovación.

Sin necesidad de profundizar más en el análisis de los signos de la innovación contamos con los datos suficientes para decir que se trata de un cambio cuyo entorno de decisión es de carácter incrementalista; un argumento más a favor de nuestra postura lo presenta la parte introductoria a la Ley de ordenación general del sistema educativo (1990) cuando se refiere a dar satisfacción a las exigencias formativas del entorno social y productivo, a la progresiva integración de nuestra sociedad en el marco Comunitario que nos sitúa ante un horizonte de competitividad, movilidad y libre circulación y, al acelerado cambio de los conocimientos, procesos culturales y productivos. En suma se justifica el cambio por las condiciones críticas de la sociedad. Es pues, un grado de cambio alto, necesitado de gran actividad innovadora, tiene como fin probar y difundir soluciones para los problemas y que necesita un alto grado de evaluación. Al cliente y a los profesionales se les han marcado ya los objetivos mediante decisiones de planeamiento políticas, y toda la actividad se concentra en parte de las decisiones de estructuración ya que algunas también están marcadas por decisiones políticas, restando por tanto las de instrumentación y recuperación o reciclaje. Todo lo cual lleva a la necesidad de adoptar tipos de evaluación que vienen determinados por los tipos de toma de decisiones que las exigen; es decir, la evaluación será mínima sobre el contexto ya que está al nivel de decisiones de planeamiento, y se distribuirá en evaluación de input, proceso y producto.

\section{INSPECCIÓN PROFESIONAL E INNOVACIÓN EDUCATIVA}

Considerando, según ha quedado patente en lo expuesto, que la inspección educativa requiere, para su ejercicio, una preparación específica por la singularidad de las funciones que desempeña en la educación, distintas de las que corresponden a otros agentes del sistema educativo, podemos afrontar algunas conclusiones sobre la viabilidad y eficacia de su intervención en la innovación, cambio o reforma educativa.

Hay que puntualizar otro aspecto, es el hecho de que dada la multiplicidad de funciones y papeles que tiene que asumir no se saque la conclusión de que las deba desempeñar simultáneamente, sino que la estrecha relación de unas funciones con otras pide esa múltiple competencia como exigencia formativa.

Lo que se puede demandar y esperar de la inspección educativa en la coyuntura a que nos referimos, tras la promulgación de la LOGSE y disposiciones complementarias de carácter innovador, depende de una serie de presupuestos y condicionamientos. Por supuesto están vinculadas a lo que acabamos de ver, es decir, la interrelación entre el 
sistema educativo, la toma de decisiones y los pasos que requiere toda innovación apoyada en la evaluación.

Entendemos que deben cumplirse dos presupuestos. La capacitación de todos los inspectores en lo conceptual, técnico y humano, y, por otra parte, la atribución expresa de autoridad formal, como complemento de la autoridad competencial previamente reconocida, supuestas las garantías del sistema de selección. Ya en el propio ejercicio de su actividad necesita: a) encontrarse inserto en la estructura de línea del sistema educativo en sus distintos niveles lo cual implica la posibilidad de promoción funcionarial; b) el ejercicio de la actividad coordinadora en las fases de desarrollo, difusión y establecimiento de la innovación; c) la posibilidad de tomar decisiones, a título individual o colegiado según convenga, en las fases de estructuración e instrumentación, con la aplicación correspondiente de normas; d) desarrollo del papel de asesoramiento en la fase de recuperación o reciclaje; e) posibilidad de ejercer función evaluadora en el input, proceso y producto del sistema.

Con los requisitos citados los inspectores podrán desempeñar los papeles que en su momento mencionamos y describimos (informador, intérprete, agente de mantenimiento, desarrollo y guía, y los de liderazgo y evaluador); todos esos papeles garantizan no solo el progresivo establecimiento de la innovación sino la posibilidad de recuperación.

Sería importante que inspectores de otros países fueran observadores de nuestro proceso innovador y pudieran aportar estudios comparados, fruto de investigaciones y experiencias precedentes.

\section{BIBLIOGRAFÍA}

AlKIN, M. C. (1972). «Accountability defined» en Evaluation Comment; Vol. 3, nº 3, pp. 1 a 5.

Buj GIMENO, A. (1985). «Supervisión educativa, retroinformación diagnóstica y toma de decisiones a multinivel». Ponencia en el Seminario sobre: Control de la calidad de la enseñanza. Madrid. USITE-ICEU (Texto ciclostilado).

Buj Gimeno, A. (1988). «Hacia un nuevo modelo de inspector profesional de educación». Comunicación al IX Congreso Nacional de Pedagogía. Actas. Caja de Ahorros provincial. Alicante.

Buj Gimeno, A. (1991). «La inspección y su papel en la reforma educativa». Ponencia en Xornadas sobre a LOGSE: A Inspección da educación ante a reforma educativa. Castelo de Soutomayor Xuño. 1991.

Glickman, C. D. (1985). Supervision of instruction. Allyn and Bacon. Boston.

Havelock, R. G. (1973). The Change Agent's Guide to Innovation in Education. Ed. Educ. Technology Publications Englewood Cliffs. New Jersey U.S.A.

JAMES, M. J. (1973). Handbook of educational supervision. A guide for the practitioner. Allyn and Bacon. Boston.

LOGSE (1990). Texto de la Ley de ordenación general del sistema educativo.

MosSER, R. (1974). Nuevo enfoque de la supervisión. Un desafio al concepto tradicional. Ed. «El Ateneo». Buenos Aires. 
PAUVert, J. C. (1986). Les personnes de supervision pèdagogique: nouvelles fonctions et formation. UNESCO. Paris.

RIVAS, M. (1984). «Modelos de inspección educativa en un sistema escolar diversificado». En Educación y sociedad plural. Actas del VIII Congreso Nacional de Pedagogía. Santiago de Compostela, 1984.

I Simposio Europeo de InsPeCcion Educativa (1986). Actas. Ed. Usite. Cea Bermúdez 14. Madrid.

II Simposio Europeo de Inspeccion Educativa (1990). Conclusiones. Conselleria de Cultura, Educació i Esports. Govern Balear. Palma de Mallorca.

STAKE, R. E. (1967). «The Countenance of Educational Evaluation». Teachers College Record, $\mathrm{n}^{\circ} 7$, pp. 523 a 540.

StufFlebeam, D. L. y otros. (1971). Educational evaluation and Decision Making. Bloomington. Phi Delta Kappa.

Stufflebeam, D. L. (1976). «Evaluating the Contex, Input, Process and Product of Education». Ponencia presentada en el Congreso Internacional de Educación Física. Helsinki.

TYLER, R. (1969). Educational Evaluation: New Roles, New Means. Sociedad Nacional de estudios sobre Educación. Chicago.

UnRUCH, A. y TURNER, H. E. (1970). Supervision for change and innovation. Hougton Mifflin. Co. Boston.

Voltes Bou, P. (1978). La teoría general de sistemas. Ed. Hispano Europea. Barcelona. 